\title{
GRAVITATIONAL COLLAPSE
}

\author{
R. PENROSE
}

University of Oxford, England

\begin{abstract}
In the standard picture of gravitational collapse to a black hole, a key role is played by the hypothesis of cosmic censorship - according to which no naked space-time singularities can result from any collapse. A precise definition of a naked singularity is given here which leads to a strong "local' version of the cosmic censorship hypothesis. This is equivalent to the proposition that a Cauchy hypersurface exits for the space-time. The principle that the surface area of a black hole can never decrease with time is presented in a new and simplified form which generalizes the earlier statements. A discussion of the relevance of recent work to the naked singularity problem is also given.
\end{abstract}

The theoretical picture of gravitational collapse to a black hole is now a familiar one (Penrose, 1969; Hawking and Ellis, 1973), so I shall dwell only briefly upon it. The essentials are depicted in Figure 1. Matter collapses inwards under the influence of gravitation until a situation is reached from which there is no escape. We may rec-

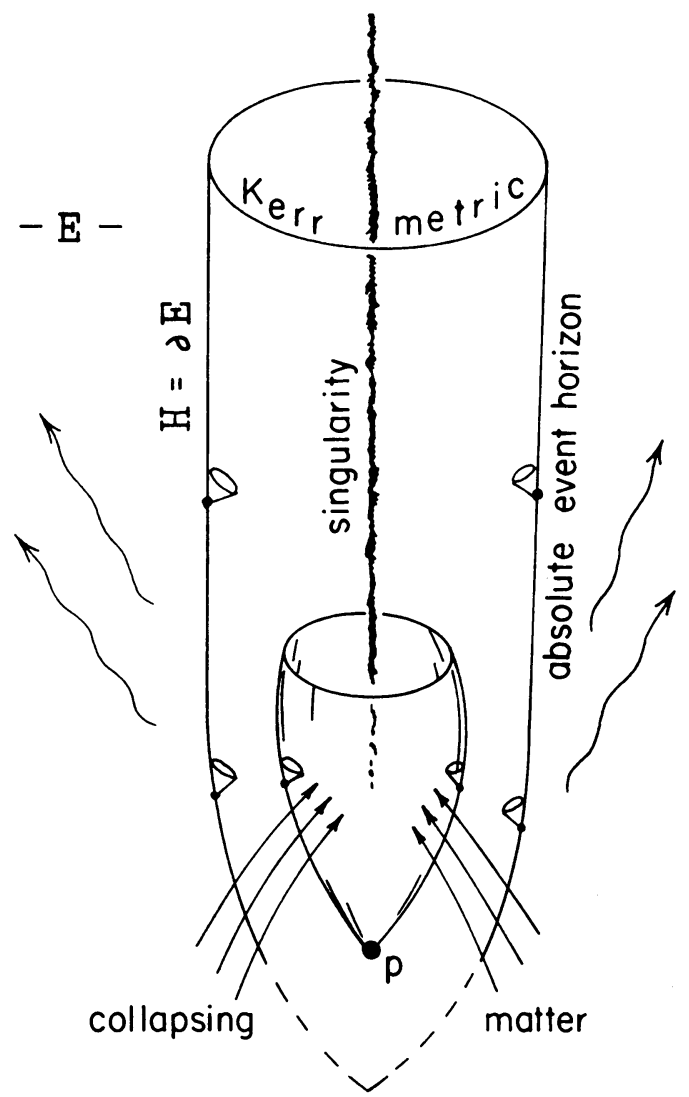

Fig. 1. Gravitational collapse to a black hole according to a standard viewpoint : the future light cone of $p$ starts to reconverge and the external field settles down to that of a Kerr black hole. 
ognise that such a situation has occurred by the presence of a trapped surface (Penrose, 1968) or, as in the case depicted, by the fact that sufficient matter crosses the future light cone of some event $p$ to cause the divergence of the light rays (null geodesics through $p$ ) to change sign, so that the rays begin to converge towards one another again. When this occurs we can invoke the Hawking-Penrose theorem (Hawking and Penrose, 1970) to deduce the existence of some form of space-time singularity. To derive this conclusion we need only assume, in addition to Einstein's equations, that the density plus each principal pressure is non-negative and the density plus the sum of the principal pressures is likewise non-negative, that there are no closed timelike curves and that the space-time is appropriately 'general' - all of which are very reasonable requirements from the physical point of view.

At this juncture the assumption of cosmic censorship can be brought in (Penrose, 1969, 1974a). This hypothesis forbids the existence of a naked singularity, i.e. any space-time singularity arising in a collapse from which a signal can originate which escapes to infinity. I shall discuss the status of this assumption a little later. For the moment I merely point out that it is the most conservative of the positions available to us. The violation of cosmic censorship would lead to a picture of gravitational collapse much more radical than that of a black hole.

Consider, now, the set $E$ consisting of all events from which a timelike curve (or a null curve - it makes little difference) can be drawn into the future to infinity. The boundary $\partial E$ of $E$ is called the absolute event horizon (Penrose, 1969). Thus the absolute event horizon may be thought of as the boundary between the regions consisting of those events from which an observer can escape to infinity and those events from which he cannot. The principle of cosmic censorship may now be adopted in the form which states that all the resulting space-time singularities must be surrounded by - and must not lie on - the absolute event horizon $H=\partial E$. One precise statement of this hypothesis is Hawking's condition of future asymptotic predictability (Hawking, 1972; Hawking and Ellis, 1973). This applies, strictly speaking, only in the case of an asymptotically flat space-time. I shall give a more general criterion shortly. When such a non-singular absolute event horizon exists we say that the universe model contains a black hole or black holes - depending upon the number of disconnected pieces into which this horizon falls. We may also envisage two or more black holes congealing into one. Then the absolute event horizon resembles the situation depicted in Figure 2. When a black hole settles down into a stationary state then, as is virtually established by the results of Israel (1967), Carter (1971), Hawking (1972), and others (Müller zum Hagen et al., 1972; cf. also Hawking and Ellis, 1973), it takes up the configuration of a Kerr metric. This is defined by just two parameters $m$ and $a$, where $m$ specifies the mass and $a m$ the angular momentum. For a black hole, $m \geqslant a$.

An important feature of black holes subject to cosmic censorship is the area principle. This states that if $S_{1}$ and $S_{2}$ are two cross-sections of $H$, where $S_{2}$ lies entirely to the future of $S_{1}$ along $H$, then the area of $S_{1}$ cannot exceed that of $S_{2}$. (Each $S_{i}$ is a spacelike 2-surface, not necessarily smooth). Hawking (1972) has used the area 


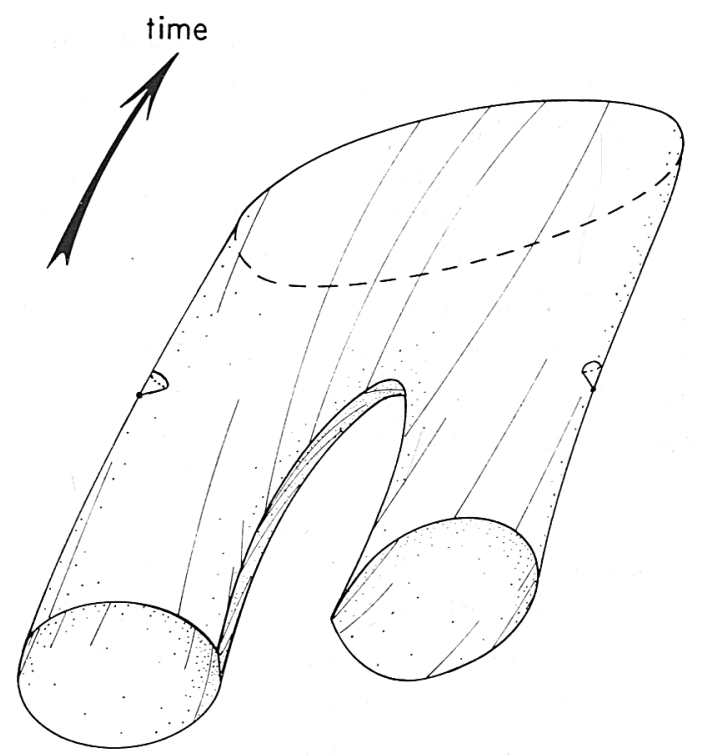

Fig. 2. Two black holes congealing to form a single black hole.

principle to derive an uuper bound to the amount of gravitational radiation emitted when two black holes coalesce. For this, the area principle is used in the form

$$
A_{1}+A_{2} \leqslant A_{3},
$$

where the subscripts 1 and 2 refer to the colliding black holes and 3 to the resultant black hole, the surface area $A_{i}$ of each black hole being given by

$$
A_{i}=8 \pi m_{i}\left(m_{i}+\left(m_{i}^{2}-a_{i}^{2}\right)^{1 / 2}\right),
$$

when the black holes are settled into their respective Kerr configurations. Thus, in this case the cross-section initially consists of two pieces and finally of one piece, but the area principle still holds.

It is worth indicating here the basic properties which lie behind the area principle. The key fact is that every point of $H$ is the past end-point of some future-endless null geodesic lying on $H$, of infinite affine extent. If $\delta A$ is an element of surface area of some spacelike cross-section of $H$ and if we propagate $\delta A$ along the null geodesic generators of $H$, we get the equation (cf. Penrose, 1968)

$$
-\frac{\mathrm{d}^{2}(\delta A)^{1 / 2}}{\mathrm{~d} v^{2}}=(\delta A)^{1 / 2}\left\{\sigma \bar{\sigma}+\Phi_{00}\right\} \geqslant 0,
$$

where $v$ is an affine parameter along the relevant null geodesic $\gamma$, where $\sigma$ is its complex shear, and $\Phi_{00}$ is minus one-half the Ricci tensor component associated with $\gamma$. The weak energy condition (energy plus each principal pressure non-negative) gives $\Phi_{00} \geqslant 0$. It follows that if $\mathrm{d}(\delta A)^{1 / 2} / \mathrm{d} v$ ever becomes negative, then $(\delta A)^{1 / 2}$ must de- 
crease to zero within a finite $v$ value. But if this were to happen, then $\gamma$ could not remain on $H$ for values of $v$ beyond this ( $H$ being the boundary of a set, namely $E$, which is its own past, cf. Penrose, 1972). This would contradict the statement that each null geodesic generator of $H$ extends into the future to arbitrarily large affine parameter values. Thus $\mathrm{d}(\delta A)^{1 / 2} / \mathrm{d} v \geqslant 0$, whence $\mathrm{d}(\delta A) / \mathrm{d} v \geqslant 0$ along $\gamma$.

This shows that the surface area increases along $\gamma$ as we proceed into the future. Furthermore $\gamma$ never leaves $H$ in future directions. But it can leave $H$ in past directions. Thus, the surface area of cross-sections of $H$ can increase into the future for two reasons, either because it increases along any given $\gamma$ or because new $\gamma$ 's emerge. (Note that in the black hole collision of Figure 2, both of these situations occur.) Neither of these situations can occur in past directions. Thus, the area principle is established provided it can be shown that null geodesics generate $H$, having infinite affine lengths into the future. In fact, it is only the condition of infinite affine length which causes any difficulty. It is this that requires a suitable statement of the cosmic censorship principle.

In order to state this principle precisely we require a definition of a space-time singularity. I shall adopt a definition which is not quite the same as that suggested by the singularity theorems, but it is more useful for present purposes. The ideas are taken from Geroch et al. (1972) and are somewhat similar to those of Seifert (1971). Let us assume, for simplicity, that the space-time is strongly causal (cf. Hawking and Ellis, 1973; Penrose, 1972). We adjoin some extra 'ideal points' to the space-time which are end-points to future-endless or to past-endless causal (i.e. timelike or null) curves. We say that two future-endless causal curves $\gamma, \gamma^{\prime}$ have the same ideal future end-point if the two curves have identical pasts (i.e. $I^{-}[\gamma]=I^{-}\left[\gamma^{\prime}\right]$ in the notation of Geroch et al. (1972). Such sets are called terminal indecomposable past sets, or TIP's for short). Similarly, two past-endless causal curves have the same ideal past end-point if they have identical futures (called TIF's). (To be able to say when a future-endless causal curve has the same future end-point as the past end-point of some past-endless causal curve would require some extra complications which I do not propose to enter into here.) These ideal points form a sort of boundary to the space-time. But the boundary points need not be singularities. They may be points at infinity. To distinguish these two possibilities we may adopt one of a number of slightly different criterea. Let us choose the simplest one here and say that the boundary point is at infinity if and only if there is a semi-infinite causal curve of infinite proper length which has that boundary point as an ideal end-point. Otherwise, we can say we have a finite boundary point. In the case that we have a space-time which is maximally extended (i.e. not a proper part of any other connected space-time), then all finite boundary points may be reasonably interpreted as singular points of the space-time. One might also consider some of the points at infinity also to be singular, but I shall not bother with this possibility here.

Let us now try to interpret the cosmic censorship hypothesis in these terms. We need a definition of a naked singularity, but we must be careful not to rule out the 'big bang' in the exclusion of naked singularities, even though the big bang singu- 
larities of the normal cosmological models are indeed naked in the sense that signals can escape from them (i.e. they are represented, in the above descriptions by past end-points of past-endless causal curves). The idea of a naked singularity is that it might arise in the collapse of ordinary matter from a non-singular initial state. I shall formalize this idea by regarding a singularity as naked if there is some observer (timelike curve) for whom the singularity lies initially to his future and subsequently to his past. This concept is in essence time-symmetrical, and it should be observed that the normal cosmological big bang does not qualify as a naked singularity in this sense. The definition is basically 'local' in that no mention is made of signals escaping to infinity. Thus, even a singularity inside a black hole might conceivably be 'naked' to some observer who is himself inside the black hole. However, in the normal picture of spherically symmetrical collapse, such a situation does not occur. There is some indication also (Simpson and Penrose, 1973) that in a generic perturbed collapse this situation still does not occur.

Now we can apply the above idea to the aforementioned definition of a singularity either of two ways around. Let us suppose, first, that we are concerned with the ideal past end-point $q$ of a past-endless timelike curve $\gamma$ (i.e. with the TIF $I^{+}[\gamma]$ ). We can say that $q$ lies to the (causal) future of a point $p$ if the future of $p$ contains the future of $\gamma$ (i.e. $\left.I^{+}(p) \supset I^{+}[\gamma]\right)$. In this case, any point $r$ of $\gamma$ lies to the future of $p$, so there are timelike curves from $p$ to $r$. An observer following one of these timelike curves will have $q$ to his future when he is at $p$ and to his past when he is at $r$. (See Figure 3.)

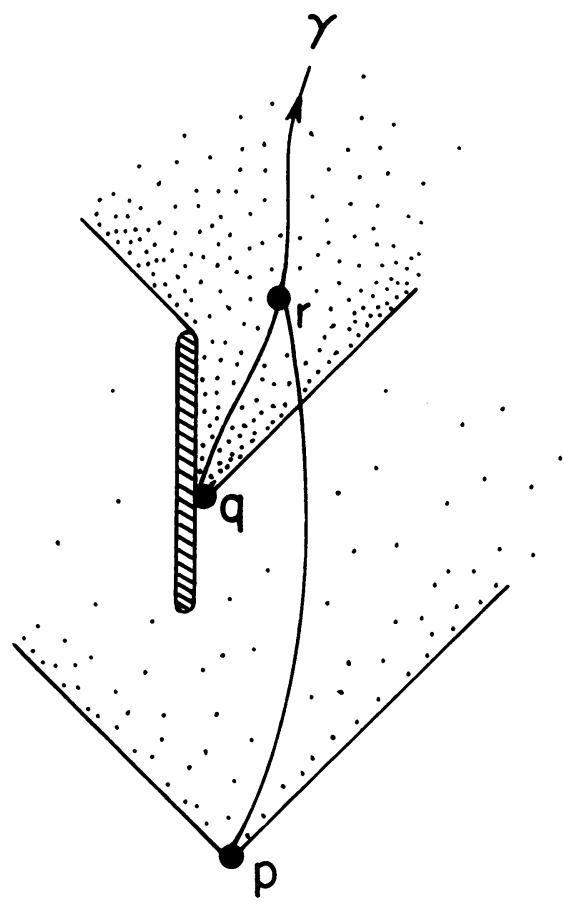

Fig. 3. A naked singularity $q$ - the future of some point $p$ contains the future of $q$. 
Thus, if $q$ is a singular point, then this is the situation where a naked singularity occurs. A similar situation might possibly arise when $q$ is a point at infinity. For purposes of the present discussion I want to regard such a possibility as something akin to a naked singularity. The normal cosmological models do not admit such an occurrence. (The complete anti-de Sitter space is an exception, cf. Penrose (1968), but cannot be regarded as a 'normal' cosmological model.) Also, while this possibility can occur in certain idealized models of collapse, there is some indication that it may be unstable in the sense that small perturbations could lead to genuine curvature singularities (cf. Simpson and Penrose, 1973). I shall refer to the hypothesis which excludes the above situation from occurring whether or not $q$ is a point at infinity as strong cosmic censorship.

We could also apply the above argument in a time-reversed sense, allowing $q$ to be the future end-point of a future-endless timelike curve $\gamma^{\prime}$. It turns out (although this is not immediately obvious) that we get precisely the same condition of strong cosmic censorship as above if we proceed in this time-reversed way, i.e. if we rule out any point $r$ whose past could contain the past of such a curve $\gamma^{\prime}$. In fact, it can be shown (cf. Penrose, 1972) that strong cosmic censorship is equivalent to Leray's condition of global hyperbolicity. Using a theorem of Geroch (1970) we can state global hyperbolicity to be the condition that a Cauchy hypersurface exists for the spacetime. Geroch's theorem also implies that the entire universe model is then topologically the product of this Cauchy hypersurface with an open timelike line. Thus, we may say that if the strong cosmic censorship principle holds, then the topology of the universe is unchanging for all time. (I shall discuss this more fully, in the case of a closed universe model (Penrose, 1974b)). In fact, global hyperbolicity leads to many other simplifications in the global structure of a space-time. For example, any two points with a timelike separation in such a space-time can be connected by a timelike geodesic which maximizes the lengths of all timelike curves which connect the points. Thus, if strong cosmic censorship turns out to be a reasonable requirement for realistic space-time models, then many global problems connected with cosmology and black-hole structure can be handled without fear of the sort of pathology arising which could complicate so much of the earlier global analysis.

As one example of the utility of strong cosmic censorship, let me give a statement of the area principle which generalizes the particular version for asymptotically flat space-times given by Hawking (1972) (cf. also Hawking and Ellis, 1973). Suppose the space-time is globally hyperbolic and the energy condition holds everywhere. Define $E$ to be the set of points which are past end-points of timelike curves of infinite length. The required result is that the absolute event horizon $\partial E$ then satisfies the area principle (i.e. if $S_{1}$ and $S_{2}$ are two sections of $\partial E$, with $S_{2}$ to the future of $S_{1}$, then the area of $S_{2}$ is not less than that of $S_{1}$ ). To specialize this result to the situation considered by Hawking, namely to a space-time which is future asymptotically predictable from some spacelike hypersurface $K$, we need consider, for our space-time manifold, only that portion of the entire space-time which is the interior of the domain of dependence of $K$ (cf. Hawking and Ellis, 1973; Penrose, 1972). But the generalized version of the 
result as stated here may be applied also to cosmological situations. I shall give a proof and discussion of the result elsewhere.

What is the theoretical evidence in favour of or against cosmic censorship (not necessarily in its strong form)? In my opinion the evidence in either direction is very scanty indeed. I think that in a collapse which does not differ very greatly in its initial conditions from the standard spherically symmetrical collapse situation I described first, then some form of cosmic censorship is likely to be valid. The analysis by various workers of the stability of the Kerr metric tends to confirm this belief. (Much of this work is being described elsewhere in this symposium (cf. Chandrasekhar, 1974; Press, 1974; Teukolsky, 1974).) It seems likely that this programme will come to a successful conclusion in the near future and that the Kerr solution (for all $m, a$ with $m>a$ ) will be declared 'officially' to be stable. Perhaps I can be forgiven, on the other hand, if I pose a few queries concerning the whole question of stability for a black hole. I am not trying to cast any doubt on the existing analysis but merely suggest some further questions that one might attempt to answer.

In the first place, it is not completely clear to me that it is legitimate to assume, in the context of establishing that all solutions settle into a Kerr configuration, that a black hole is ever exactly stationary. I have in mind the situation which arose in connection with the Newmann-Penrose constants for asymptotically flat space-times. In fact, one can show quite rigorously that a transition from one exactly stationary state to another exactly stationary state via an intermediate phase in which gravitational radiation is emitted cannot occur unless a certain combination of multipole moments returns to its original value (Newman and Penrose, 1968). But this does not mean that this multipole moment combination must return to its original value in order for the system to settle down. It is simply that the presence of a small and ever-decreasing disturbance in the distant gravitational field (the backscattered gravitational radiation) is always just sufficient to prevent any inference concerning the final multipole moment combination to be drawn. I think it is unlikely that anything of this nature could arise to spoil the black hole results, but the issue is not clear to my mind.

It is also not quite clear to me whether the perturbation analysis is actually aimed at excluding the possibility that the absolute event horizon might itself develop into a curvature singularity - which would then be a naked singularity. A 'perturbation' which is actually singular on the event horizon might be excluded on the basis that it represents unreasonable initial data with which to start, but this becomes less clear if the black hole is never assumed to have quite reached a stationary state.

These points are perhaps subtleties and may well have no real significance for the question of black hole stability. However, a more serious question concerns the stability of a black hole near the critical case $a=m$. If $a$ is only marginally smaller than $m$ and a perturbation is applied which is in some sense comparable with the difference $m-a$, then it is by no means clear that such a perturbation can be regarded as small. It is difficult to see how this sort of disturbance could be analyzed within the framework of perturbation theory. 
Of course, the whole question of large disturbances applied to black holes is quite an open one. For example, it is often assumed that if two black holes of comparable mass are brought together, then the result will again be a black hole. While I would agree that this is certainly one clear possibility, it is by no means obvious that it is the only one. The possibility that two black holes might collide to form a naked singularity is excluded only by the pure assumption that the cosmic censorship hypothesis holds. This would again be a question of an absolute event horizon developing into a naked singularity - a circumstance which I do not see how to exclude on theoretical grounds. Perhaps the computer analysis by DeWitt and his co-workers of a black hole head-on collision will shed some light on this question. It is also possible that two black holes spiralling into one another might produce a qualitatively different result from a head-on collision.

In view of the above uncertainties it is worthwhile to investigate whether or not it is possible to set up initial states of collapse for which a contradiction with the standard picture might be obtained. The combination of area principle, mass-energy conservation, and the final situation of a Kerr metric, together impose definite constraints on the initial geometry. Basically, one is not allowed an initial situation involving a trapped surface of too great area for the initial mass involved. I have described elsewhere (Penrose, 1974a) an attempt at obtaining a contradiction with the standard picture (and hence with cosmic censorship) in this way. Some partial results by Gibbons (1973) have made it seem unlikely that a contradiction with the standard picture can be arrived at by such considerations alone.

A more direct attack on the naked singularity question is that of Müller zum Hagen et al. (1973). They construct an explicit solution of Einstein's equations which describes a collapsing dust cloud which eventually forms a black hole. However, before doing so, the dust encounters caustics at which the density becomes infinite. For a short while this region of infinite density - and therefore infinite curvature - is visible from infinity (Figure 4). Thus the solution must be said genuinely to describe a collapse with a naked singularity. It seems that the solution should be stable under small perturbations of the initial state since Grischuk (1967) has shown that fully general timelike singularities (involving rotation) can occur with dust. Müller zum Hagen et al. (1973) also show that in the spherically symmetrical case, the introduction of a bounded pressure does not substantially affect their conclusions: a naked singularity can still arise. Stability of the singularity situation for such a fluid under general perturbations (which involve some rotation) is not considered however.

I think that these examples are interesting more for the questions that they raise than for the questions that they answer. I certainly do not feel that in themselves they overthrow the cosmic censorships principle, but they do cause one to wonder what form of precise statement such a principle should have, if one is to have any hope of proving it. Naked singularities which are not stable under perturbations of the initial conditions should presumably be discounted. But what about perturbations of the equations of state? What kinds of such perturbation should be permitted? Or would it be simpler to restrict attention just to the vacuum case? What role should be played 
by considerations such as those of Hagedorn (1968) according to which the maximum permissible pressure-to-density ratio goes down to zero as the density increases to infinity? If this is valid, the material would behave more and more like dust as the density increases.

Finally, we should ask what is the observational status of naked singularities.

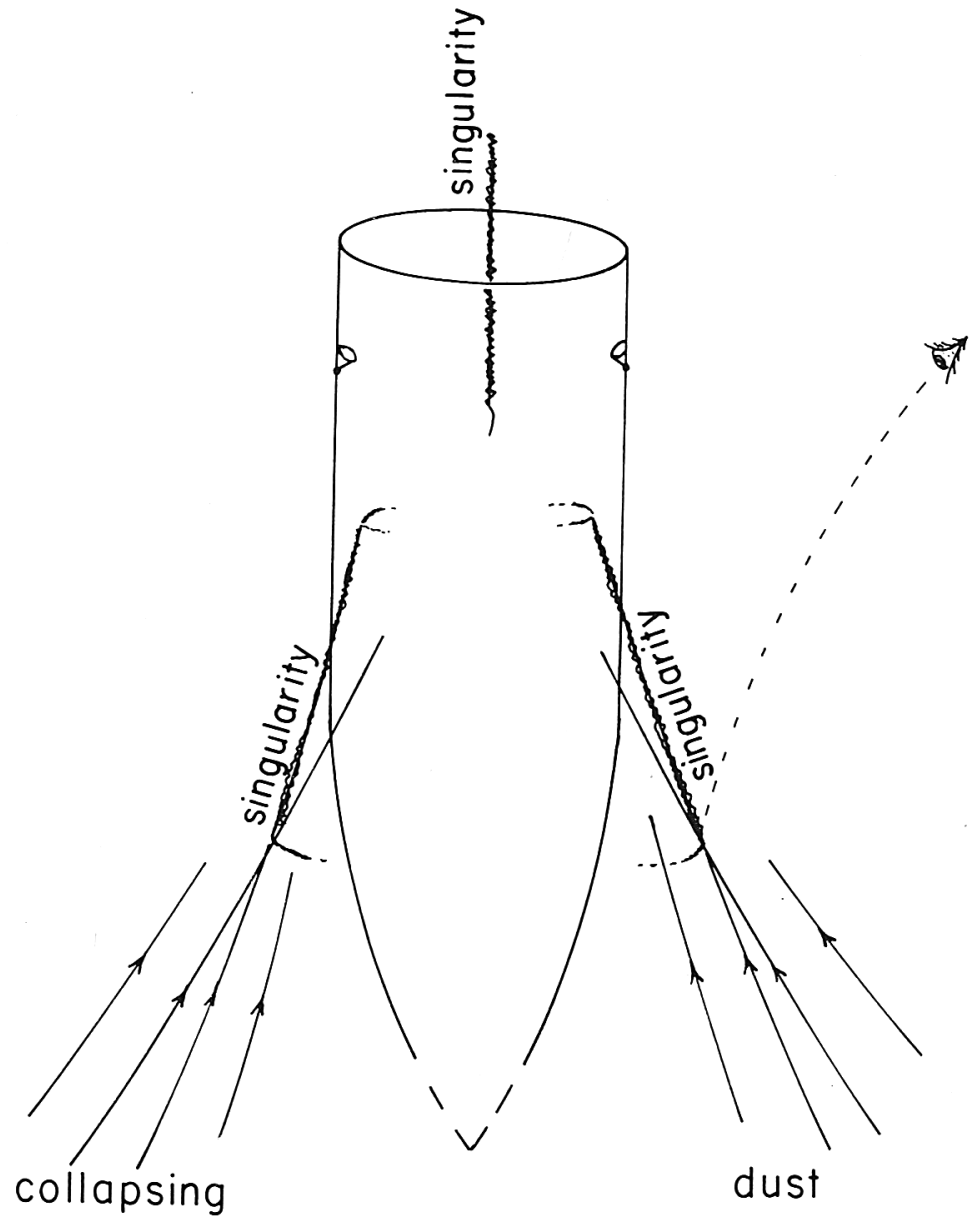

Fig. 4. The Müller zum Hagen-Seifert-Yodzis model of a collapsing dust cloud with naked singularity of the 'shell-crossing' type.

There are certainly many strange phenomena observed to occur, especially in galactic nuclei and in quasars. The temptation to invoke some sort of naked singularity as an explanation must be strong. But the reasons for doing so would seem to be of a rather negative character. The physics at a naked singularity would be largely unknown, so explanations of this kind would have little predictive power. I feel that there is one possible exception, however, and this is in Weber's observations. If his results turn out to be substantiated, then we shall be faced with a problem of energy balance which seems to have little hope of solution unless a very effective beaming mechanism can be 
suggested which sends the gravitational waves out closely in the plane of the galaxy. Misner's ingenious suggestion (cf. Misner, 1974) for doing this by means of gravitational synchrotron radiation from particles orbiting a black hole seems now not to be feasible. There is an alternative possibility whereby a naked Kerr ring singularity achieves such beaming (Penrose, 1974a) and this should also be considered. But only if the observations pointed clearly to the necessity of such an explanation could one be expected to take such suggestions very seriously.

\section{References}

Carter, B.: 1971, Phys. Rev. Letters 26, 331.

Chandrasekhar, S.: 1974, this volume, p. 63.

Geroch, R.: 1970, J. Math. Phys. 11, 437.

Geroch, R., Kronheimer, E. H., and Penrose, R.: 1972, Proc. Roy. Soc. London A327, 545.

Gibbons, G.: 1973, to appear.

Grischuk, L. P.: 1967, Sov. Phys. J.E.T.P. 24, 320.

Hagerdorn, R.: 1968, Nuovo Cimento 56A, 1027.

Hawking, S. W.: 1972, Comm. Math. Phys. 25, 152.

Hawking, S. W. and Ellis, G. F. R.: 1973, The Large Scale Structure of Space-Time, Cambridge Univ. Press.

Hawking, S. W. and Penrose, R.: 1970, Proc. Roy. Soc. London A314, 529.

Israel, W.: 1967, Phys. Rev. 164, 1776.

Misner, C. W.: 1974, this volume, p. 3.

Müller zum Hagen, H., Robinson, D. C., and Seifert, H.-J.: 1972, Gen. Relativity and Gravitation 4, $1,53$.

Müller zum Hagen, H., Seifert, H.-J., and Yodzis, P.: 1973, Comm. Math. Phys. 34, 135.

Newman, E. T. and Penrose, R.: 1968, Proc. Roy. Soc. London A305, 175.

Penrose, R.: 1968, in C. M. De Witt and J. A. Wheeler (eds.), Battelle-Rencontres, Benjamin, New York.

Penrose, R.: 1969, Rivista del Nuovo Cimento, Ser. 1, 1, Num. Spec. 252.

Penrose, R.: 1972, Techniques of Differential Topology in Relativity, S.I.A.M., Philadelphia.

Penrose, R.: 1974a, Ann., N.Y. Acad. Sci. 224, 125.

Penrose, R.: 1974b, in M. S. Longair (ed.), 'Confrontation of Cosmological Theories with Observational Data', IAU Symp. 63, in press.

Press, W. H.: 1974, this volume, p. 93.

Seifert, H.-J.: 1971, Gen. Relativity Gravitation 1, 247.

Simpson, M. and Penrose, R.: 1973, Int. J. Theor. Phys. 7, 183.

Teukolsky, S.: 1974, this volume, p. 92. 